$\begin{array}{ll}\text { Balkanologie } & \begin{array}{l}\text { Balkanologie } \\ \text { Revue d'études pluridisciplinaires }\end{array} \\ & \begin{array}{l}\text { Vol. } \mathbf{1 5} \mathrm{n}^{\circ} \mathbf{1} \mid \mathbf{2 0 2 0} \\ \text { Mémoires performatives : faire des passés et des } \\ \text { présents }\end{array}\end{array}$

\title{
Introduction. Mémoires performatives : faire des passés et des présents
}

Introduction. Performing Memory: Doing Past and Present

\section{Bianca Botea et Vintilă Mihăilescu}

\section{OpenEdition \\ Journals}

\section{Édition électronique}

URL : https://journals.openedition.org/balkanologie/2470

DOI : $10.4000 /$ balkanologie. 2470

ISSN : 1965-0582

Éditeur

Association française d'études sur les Balkans (Afebalk)

Référence électronique

Bianca Botea et Vintilă Mihăilescu, «Introduction. Mémoires performatives : faire des passés et des présents », Balkanologie [En ligne], Vol. $15 n^{\circ} 1$ | 2020, mis en ligne le 01 juin 2020, consulté le 05 août 2021. URL : http://journals.openedition.org/balkanologie/2470 ; DOI : https://doi.org/10.4000/ balkanologie. 2470

Ce document a été généré automatiquement le 5 août 2021

(c) Tous droits réservés 


\title{
Introduction. Mémoires performatives : faire des passés et des présents
}

Introduction. Performing Memory: Doing Past and Present

\author{
Bianca Botea et Vintilă Mihăilescu
}

1 Ce numéro thématique porte sur les processus mémoriels dans les sociétés balkaniques contemporaines, abordés à partir de leur dimension performative.

2 La création d'un champ de recherche transdisciplinaire autour des questions mémorielles, ou memory studies, est relativement récente puisqu'on peut la faire remonter aux années $1990^{1}$. Le chemin vers la reconnaissance et la réhabilitation de la mémoire par rapport à l'histoire, et sa prise en compte par les historiens, est lui-même un processus tardif intervenant, dans les sociétés occidentales, vers la fin des années 1970 et au début des années $1980^{2}$.

Dans les pays des Balkans, l'arrivée des études sur la mémoire est encore plus récente. Comme le montre Vintilă Mihăilescu pour le cas de la Roumanie, l'intérêt pour cette question s'est tout d'abord développé dans un contexte général de déconstruction du discours nationaliste, déconstruction à laquelle les sciences sociales, et en particulier

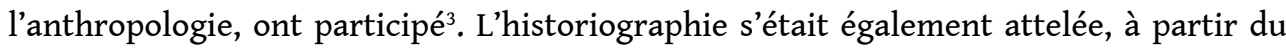
milieu des années 1990, à une déconstruction des "grandes narrations nationales" allant de pair avec un intérêt plus ciblé pour les narrations historiques régionales ou locales. Dans ce même mouvement s'inscrit, comme le note l'auteur, une mutation méthodologique en rapport avec un changement d'échelle. Celle-ci entraîne une importance accrue accordée au recueil des « histoires de vie » des gens ordinaires, à la dimension subjective et localisée des expériences des individus, ainsi qu'aux études sur la mémoire sociale. Il s'agit, plus largement, d'un déplacement de l'accent, depuis les discours de l'ethnogenèse millénaire et holiste - construite dans le sillage du folklorisme - vers une analyse des dynamiques ethniques locales et régionales, témoins de processus de différenciation culturelle et sociale. 
$4 \quad$ Un autre contexte important à rappeler afin de situer l'émergence des préoccupations scientifiques concernant les questions mémorielles est le fleurissement, lors de la première décennie après la chute du communisme, des écrits autobiographiques et mémoriels. La libération de la mémoire, désormais affranchie de la censure qui fut imposée ou auto-imposée durant le communisme, à des degrés différents selon les pays, et la démocratisation du droit à la mémoire se sont exprimées par un besoin pressant de se rappeler et d'offrir des témoignages ${ }^{4}$. Les scientifiques ont accompagné et intensifié à leur tour ces préoccupations de la société pour la mémoire, préoccupations qui se sont traduites, en dehors des écrits autobiographiques, par la création de lieux et d'institutions mémoriels (notamment pour commémorer les victimes des déportations et, plus largement, des politiques répressives des régimes communistes).

5 Ces tendances locales, régionales et nationales s'inscrivent dans un phénomène plus général de "mondialisation de la mémoire ", comme le rappelle Henry Rousso ${ }^{5}$, un « phénomène planétaire de réactivation du passé » qui démarre dans les années 1990 et « tend à susciter des formes de représentations collectives et d'actions publiques qui se ressemblent ». La nouveauté de ce phénomène, comme le note l'auteur, ne tiendrait pas à

[...] l'existence de récits historiques ou de mémoires singulières fondées sur la langue, l'ethnie, la région ou une expérience partagée, qui sont une réalité de toujours, notamment dans des aires où les systèmes politiques et sociaux accordent une place importante sinon des droits équivalents aux minorités, qu'à la formation d'un nouvel espace public, au niveau national, régional (notamment européen) et mondial 6 .

6 Ce phénomène mémoriel mondialisé est caractérisé par une prise de parole plus active d'acteurs divers proposant une pluralité d'interprétations et des narrations historiques différentes du seul discours national construit par des instances classiques de production de la connaissance historique. Les discours du scientifique et du politique sont doublés par les discours des militants, des amateurs et d'autres acteurs participant à ce processus. Pensons par exemple, dans plusieurs pays balkaniques, aux promenades mémorielles et aux circuits autour des lieux de la mémoire du communisme organisés à l'initiative de différents amateurs, associations ou petites entreprises locales d'action culturelle ou touristique.

7 Une autre dimension caractéristique du nouvel espace public dont parle H. Rousso serait la montée en puissance de la figure de la victime. Nous serions moins à l'«ère du témoin ", qu'à l'« ère de la victime » et de la réparation7. Dans ce contexte, la mémoire serait devenue une valeur positive quasi universelle, à l'opposé de l'oubli considéré comme une catégorie négative. Un bémol devrait néanmoins être mis ici comme le cas des sociétés balkaniques nous le montre bien. Nous pouvons par exemple constater que les différents types de mémoire ne bénéficient pas du même degré de valorisation et que, dans ces pays, des champs entiers ou des secteurs de la mémoire, comme la mémoire de l'expérience ouvrière, industrielle ou du quotidien de la période communiste, ne sont pas mobilisées, symbole parfois de ce que justement nous ne devrions pas nous rappeler.

Dans ce contexte à la fois global et spécifique à chaque pays, région ou réalité locale, le champ d'études sur la mémoire dans les Balkans s'est développé, comme partout ailleurs, par un déploiement sur un paysage thématique de plus en plus vaste et varié : des mémoires des guerres, des déportations et des événements traumatiques aux mémoires de l'expérience quotidienne durant le régime communiste, en passant par les 
mémoires mobilisées dans les processus de nationalisme, de construction des territoires ou des frontières, jusqu'aux aux mémoires urbaines et industrielles, au tourisme mémoriel et aux mémoires du corps, rien ne semble échapper à la thématique mémorielle ${ }^{8}$.

Ce numéro n'a pas pour but d'ajouter des thématiques et domaines nouveaux à cette liste déjà longue de travaux sur la mémoire; il s'agit plutôt de considérer la multiplication des pratiques mémorielles et la mise au travail du passé à partir de quelques « scènes » contemporaines où se construit ce qui a valeur de mémoire, c'est-àdire un sens partagé sur ce qui est (ou devrait être) considéré comme mémoire. Dans ce sens, l'objectif principal est moins de traiter des usages de la mémoire, de ses enjeux ou des arènes mémorielles à l'œuvre dans les sociétés balkaniques, une dimension largement abordée dans les travaux existants. Même si elle sera présente de manière inhérente dans les articles de ce numéro, choisir cette perspective comme point de départ présenterait néanmoins un écueil, comme le montraient Michel Peroni et Dominique Belkis dans un numéro de revue consacré à la «Pragmatique de la mémoire ${ }^{9}$ » l'approche des usages, des enjeux et des cadres sociaux de la mémoire suppose implicitement que les mémoires sont déjà là en tant qu'objet déjà formulé et constitué, soumis ultérieurement à des négociations et à des mises en partage. La perspective des mémoires performatives qui nous intéresse ici suppose au contraire d'aborder le phénomène mémoriel en amont, par les scènes, les dispositifs et les mécanismes qui font advenir ce qui fait mémoire ou ce qui a valeur de mémoire et de "passé ", en tant que contenu mémoriel partagé et continuellement soumis à des remises en question et à des créations.

Dans ce texte d'introduction, nous poserons dans un premier temps quelques repères pour une approche des mémoires performatives qui nous intéresse ici. Nous donnerons ensuite un aperçu des articles de ce numéro et de la manière dont ils mettent en œuvre cette approche.

\section{Repères pour une approche des mémoires performatives}

11 Depuis les travaux pionniers de sociologie de la mémoire de Maurice Halbwachs, nous savons que la mémoire n'est pas un « bagage » informationnel qu'un individu détient et qu'il transmet tel quel aux générations futures dans une continuité qui va de soi. En outre, pour M. Halbwachs, la mémoire n'est pas un phénomène proprement individuel car « on ne souvient jamais seul» et le processus de remémoration et de transmission nécessite des «cadres sociaux» qui rendent possible sa réalisation ${ }^{10}$. Dans cette perspective, la mémoire est un processus actif, une construction à partir d'une matérialité ou d'un contexte social et des actions menées dans le présent.

Sur un autre plan, dans son approche de la tradition, Jean Pouillon rappelle qu'elle est une "filiation inversée " : c'est le fils qui engendre le père et il peut ainsi se permettre de s'en offrir plusieurs ${ }^{11}$. Nous pouvons dire que la «tradition» est une forme de mémoire sociale parmi d'autres, mettant en scène des passés "à la carte " selon les goûts et intérêts du présent. À l'inverse des mémoires récupératrices se trouvent les mémoires constructives, qui ne mettent plus en scène le passé légitimé par le présent, mais un présent légitimé par le passé. Ce ne sont là que les deux visages des mémoires 
performatives, qui, à la fois, font un recours électif et produisent ce qui fait valeur de « passé » et de " présent ».

13 La catégorie du " performatif » doit beaucoup à John Austin ${ }^{12}$. En partant d'une analyse des énonciations performatives, Austin montre que le langage n'a pas seulement la fonction de "dire » mais aussi de "faire ». "Quand le prêtre ou le représentant de l'État me demande : 'prenez-vous cette femme pour légitime épouse ?' et que je réponds 'oui, je le veux', je ne décris rien ", selon Austin, « je fais quelque chose : je ne fais pas le reportage d'un mariage : je me marie ${ }^{13}$. Ces énoncés performatifs sont caractérisés par le fait qu'ils ne décrivent pas un état de choses, mais qu'ils sont censés accomplir l'action qu'ils désignent (« quand dire c'est faire »). En bref, l'apport de John Austin est avant tout de donner au "performatif» (par l'exemple du langage) une dimension active et créative.

14 Au-delà des travaux d'Austin, un "tournant performatif » s'affirme et se généralise dans les sciences sociales et humaines dans les années 1990. Il offre des lectures intéressantes dans des domaines et des champs variés comme l'étude de la perception, du genre, des médias, des classes, etc. ${ }^{14}$. Dans l'approche performative du genre et de la sexualité, Judith Butler ${ }^{15}$ met en avant l'idée qu'un individu n'acquiert pas une identité de genre par ce qu'il est mais par ce qu'il fait; nous devenons des hommes et des femmes par des actes répétés qui font appel à des normes sociales et culturelles et les reproduisent. Le performatif fait ici référence à ces actes répétés et normés qui vont instituer une qualité et une identité de genre. Cette dimension performative est sousentendue dans de nombreux travaux, même si parfois la notion de performatif n'est pas directement évoquée. Dans l'approche de l'espace et de la perception qui est celle de Michel de Certeau, ces deux phénomènes sont le résultat d'un processus performatif réalisé par le corps en mouvement : c'est en marchant que l'on crée et que l'on perçoit l'espace ${ }^{16}$.

15 Une contribution essentielle à l'approche du performatif a été fournie par les performance studies et par la cultural theory. Ces perspectives ont mis en avant les contenus et les formes culturelles qui circulent et se transmettent dans les gestes, le langage, les chansons, les danses, les rites et rituels, qui sont tous autant de performances. Comme le montre Joseph Roach, le paradoxe de la performance réside dans l'idée même de répétition, laquelle est en réalité une création : « aucune action ou séquence d'action ne peut être performée deux fois de la même manière. Elles doivent être recréées et réinventées à chaque répétition. Dans cet espace comportemental d'improvisation, la mémoire elle-même se révèle comme imagination ${ }^{17}$ ".

Par conséquent, le lien entre répétition et innovation est au cœur de la notion de performatif: c'est la génération du nouveau par le simple fait de "faire» et de répéter ${ }^{18}$.

17 Comme on le voit, l'approche performative a donné lieu à des lectures dans différentes disciplines et par rapport à des objets de recherche très variés. Il ne s'agit pas ici d'en offrir une analyse exhaustive, mais de prendre la perspective performative comme fil rouge pour lire les phénomènes mémoriels.

Différentes contributions importantes dans le champ de la mémoire ont ouvert le chemin dans ce sens, bien qu'elles n'utilisent pas toutes le terme "performatif » et qu'elles ne se revendiquent pas d'une telle approche. Nous avons déjà mentionné les travaux de Maurice Halbwachs sur la mémoire et ceux de Jean Pouillon sur la tradition. 
Les deux perspectives suggèrent l'idée que la mémoire est une (re)création à partir du présent.

Pour faire référence à d'autres auteurs, Paul Ricœur met en lumière les deux opérations "indivisément cognitive et pragmatique» de tout phénomène mémoriel: "'Se souvenir' c'est non seulement accueillir, recevoir une image du passé, c'est aussi la chercher, 'faire' quelque chose. Le verbe 'se souvenir' double le substantif 'souvenir'. Ce que le verbe désigne, c'est le fait que la mémoire est 'exercée' ${ }^{19}$ ». La mémoire n'est donc pas seulement le surgissement mental des images et des contenus cognitifs, elle est une activité, une action en soi. Nous pouvons par conséquent nous demander ce que cette pratique de la mémoire et le fait de la « chercher » font à la mémoire elle-même et aux contextes sociaux dans lesquels elle circule.

Joël Candau, de son côté, attire l'attention sur la confusion récurrente entre la mémoire, c'est-à-dire les souvenirs réels qu'un individu aurait gardés d'un événement, et la métamémoire, c'est-à-dire ce que nous disons et pensons être notre mémoire de cet évènement. La métamémoire est un discours sur la mémoire et sur ce qu'elle doit être, autrement dit elle est une mémoire revendiquée ${ }^{20}$. Nous pouvons notamment faire le constat de ce passage subtil des mémoires vers les métamémoires dans différentes cérémonies et pratiques de remémoration, un passage qui implique de manière inhérente un processus de (re)création du contenu mémoriel.

21 Une perspective plus explicitement ancrée dans une approche performative est proposée par Michel Peroni et Dominique Belkis à propos de «la pragmatique de la mémoire et $[\mathrm{d}] \mathrm{es}$ régimes de mémorialitée ${ }^{21} »$. Les auteurs notent que :

L'apport considérable que l'on pourrait tirer de perspectives pragmatiques et pragmatistes, aussi variées soient-elles, consiste à saisir le phénomène mémoriel non comme un objet déjà constitué dont il s'agirait d'examiner les usages ou comme un phénomène réduit à son indexation à un arrière-monde (cadres sociaux, structures sociales, champs de force...), mais comme quelque chose qui se détermine, se spécifie dans le cours d'activités pratiques en train de se faire.

Cette perspective postule que :

[...] nous ne savons pas a priori ce qu'il peut en être de cette entité [la mémoire] puisqu'il s'agit précisément d'interroger la manière, pour une part idiomatique, dont elle est instanciée dans des contextes variés. (...) Ainsi conviendrait-il de parler non de "mémoire » comme de l'objet à traiter par l'enquête sociologique, mais de "mémorialité » comme de ce avec quoi sont aux prises les acteurs ou instances engagés dans des entreprises mémorielles, ce qu'il leur appartient de spécifier et d'instruire par l'enquête, de faire tenir et de rendre sensible par des agencements pratiques.

23 L'intérêt que nous trouvons à cette perspective est double : d'une part, elle repose sur une approche pragmatiste de la mémoire, défaite de tout habillage essentialiste; d'autre part, elle propose une conception de l'enquête de terrain sur la mémoire ellemême pragmatiste. Selon ces auteurs, le travail du chercheur devient ainsi d'identifier et d'analyser les pratiques et les dispositifs mémoriels comme des formes d'enquête (à la John Dewey) menée par les acteurs eux-mêmes à la recherche d'une formulation de ce qui est ou de ce qui fait mémoire.

24 D'autres contributions mettent en lumière des aspects différents de ceux déjà mentionnés et qui se situent au cœur des mécanismes performatifs. C'est le cas de la dimension corporelle et des routines instituées par le corps. Dans son livre How Societies Remember, Paul Connerton note : 
[...] s'il y a quelque chose qu'on peut appeler mémoire sociale, c'est probablement dans les cérémonies commémoratives qu'on va la trouver; mais les cérémonies commémoratives se trouvent être commémoratives seulement dans la mesure où elles sont performatives ; la performativité ne peut pas être conçue sans un concept d'habitude ; et l'habitude ne peut pas être pensée sans une notion du corps ${ }^{22}$.

Cette dimension du corps donne, par ailleurs, une force performative plus importante à la mémoire par rapport à l'histoire. La première implique des canaux et des formes de transmission plus appropriés à l'expérience esthétique et collective, créant plus facilement des contenus et des émotions partagées plus aisément ouvertes à des (re)créations. C'est l'idée mise en avant par certains travaux dans le champ des performance studies et de la cultural theory:

La performance met en lumière la distinction entre mémoire sociale et histoire comme formes différentes de transmission culturelle à travers le temps: la mémoire requiert une participation collective, que ce soit lors d'événements théâtraux, de rituels chamaniques ou de cérémonies d'ouverture olympiques; l'histoire implique l'interprétation critique (et apparemment solitaire) des documents écrits. Les deux fonctionnent comme des formes d'oubli : les cultures sélectionnent ce qu'elles transmettent à travers la mémoire et l'histoire ${ }^{23}$.

Dans le champ artistique et de l'action culturelle, d'autres travaux analysent l'art et la culture populaire comme des lieux de formation et de circulation de la mémoire et posent explicitement la notion de mémoire performative (performing memory) ${ }^{24}$. Ces travaux explorent «les modes par lesquels les pratiques de l'art et de la culture populaire constituent des actes performatifs de mémoire générant une expérience $d u$ passé dans le présent. (...) L'art et la culture populaire mettent en acte la mémoire et génèrent des processus de mémoire. (...) Cette mise en acte est toujours performative : son contenu est renouvelé par cet acte même de remémoration ${ }^{25}$ \%. La mémoire est, dans ce sens, une performance : elle est à la fois un processus et son résultat. Selon Liedeke Plate et Anneke Smelik, la dimension créative qui est au centre de la notion de mémoire performative permet d'aller au-delà de l'idée de la mémoire comme représentation du passé (comme trace du passé dans le présent) pour la considérer plutôt comme une re-présentation dans le sens de performance ${ }^{26}$.

Enfin, alors que la force performative de la mémoire repose sur des gestes répétitifs, des routines corporelles et sur l'infiniment petit, son impact est celui d'un geste fort, voire d'une intervention politique. Pouvant être appliqué à tout, "d'une pièce de théâtre à une guerre ou un repas (...), le performatif est un acte culturel, une perspective critique et une intervention politique ${ }^{27} »$. Suivant ce propos et de retour à Austin, nous pourrons par exemple affirmer ceci : si je mets en scène un spectacle ou un musée mémoriel, je ne fais pas un reportage sur la mémoire, je "fais » mémoire (dans le double sens, de "se rappeler » et de formuler et de fixer ce qui a valeur de mémoire). C'est dans cette perspective que nous proposons de lire les pratiques observées par les contributeurs et contributrices de ce numéro autour des commémorations nationales, des spectacles de rue autour de la cuisine, des images photographiques ou des récits mémoriels.

Les pistes de réflexion exposées ici nous incitent en effet à une lecture performative des phénomènes mémoriels rencontrés dans les sociétés balkaniques. L'objectif est, d'une part, de comprendre les scènes et les dispositifs à travers lesquels se formule aujourd'hui ce qui fait mémoire dans ces sociétés; d'autre part, de regarder ce que ces 
lieux et pratiques de travail sur la "mémorialité » font à ces sociétés mêmes, aux milieux où ils se déploient et à la transformation de ces milieux.

Deux aspects en particulier nous paraissent importants ici et nous pouvons les retrouver de manière transversale dans les contributions de ce numéro. Tout d'abord, puisque comprendre ce qui a valeur de mémoire dans les sociétés balkaniques, c'est comprendre comment des mémoires individuelles ou sociales ${ }^{28}$ deviennent (occasionnellement) des mémoires partagées ou collectives ${ }^{29}$, il convient d'identifier les pratiques et les mécanismes par lesquels ce processus se réalise. La dimension performative joue un rôle central ici pour passer de l'individuel au social voire au collectif, comme nous l'avons vu entre autres avec Paul Connerton. Christoph Wulf mentionne de son côté trois mécanismes principaux dans les pratiques performatives qui constituent la culture. Ces trois mécanismes sont le langage, l'image et le corps ${ }^{30}$. Ils sont tous trois présents dans les pratiques décrites par les articles de ce numéro. La question de l'image constitue le cœur de l'article de Gilles de Rapper, qui nous donne à voir un des "lieux» de construction des mémoires du communisme dans l'Albanie contemporaine, celui des images photographiques. La performativité de la mémoire via le langage est visible dans les écrits des moines en rapport avec la labellisation du Mont Athos par l'Unesco (Isabelle Dépret), dans des récits sur le passé suscités par l'enquête de terrain au sein de la communauté rome musulmane de Dobroudja (Adriana Cupcea) ou dans les discours médiatiques et dans la presse magyarophone en Transylvanie (Biborka Ádám). Enfin, la question du corps est présente dans les rhétoriques autour des produits alimentaires «traditionnels » et dans leurs spectacles urbains composant un espace public performatif d'une mémoire sociale du goût (Vintilă Mihăilescu).

Au-delà de cette interrogation sur la possibilité de faire advenir des mémoires partagées ou collectives, un second axe de réflexion porte sur le rapport au temps qui se définit à l'occasion de ces pratiques mémorielles. Ce rapport au temps devient un analyseur intéressant pour la relation que les individus entretiennent avec le changement dans les sociétés balkaniques. Dans ce sens, les scènes performatives de la mémoire dont il est question dans ce numéro (patrimonialisation, photographie, commémorations, festivités urbaines, histoire orale et dispositif scientifique) nous apparaissent comme des lieux où s'expriment non seulement un rapport au passé, mais un rapport au présent et une relation entre passé et présent. Comme d'autres auteurs l'ont déjà mis en avant (Halbwachs, Boym ou Todorova et Gille ${ }^{31}$, convoqués dans plusieurs contributions de ce numéro), la mémoire se construit à partir du présent et la nostalgie n'est pas un simple rapport au passé, mais elle relève d'une difficulté de vivre dans le présent et d'une impossibilité de penser l'avenir. L'article d'Adriana Cupcea montre comment des mémoires relatives à la vie quotidienne des Roms musulmans durant le communisme émergent dans le contexte des difficultés de vie d'après 1989 et des changements auxquels ses interlocuteurs de la communauté rom musulmane sont confrontés, notamment un fort déclassement social et économique. Il est donc question ici non seulement de la valeur accordée au "passé », mais d'un rapport au "présent » et à l'« avenir ».

31 Si ces perspectives réitèrent l'idée que la mémoire se fabrique à partir du présent, le présent ne doit pas pour autant être érigé en repère fixe ni en catégorie stable à partir desquels on construirait des passés fluctuants ou «à la carte». Michèle Leclerc-Olive montre que le rapport au passé, mais aussi au présent, se modifie continuellement par un rapport réflexif des individus avec leur propre histoire et au fur et à mesure que se 
déroulent des nouveaux événements ou une nouvelle période significative dans leur $v^{2} e^{32}$. C'est aussi l'idée que le présent (et non seulement le futur) est un projet toujours en train de se faire et que les mémoires sont un outil pour ce projet, comme le notait déjà Paul Ricœur ${ }^{33}$. Dans son article sur la photographie en Albanie, Gilles de Rapper montre comment cet outil sert dans les années du communisme à construire la « nouvelle société » projetée par le régime communiste. Le " présent » est ici à faire et la photographie sert à ce projet, par exemple en choisissant surtout les images qui montraient les transformations urbaines et la modernisation de la société ou celles qui exaltaient l'activisme et l'idée de collectif. Aujourd'hui, plusieurs décennies après la chute du communisme, la réalisation de ces albums à partir d'images de l'époque répond à d'autres projets et s'adresse à d'autres publics, par exemple celui de documenter une époque révolue pour la mettre à distance du présent ou de faire mémoire pour les nouvelles générations qui n'ont pas connu cette époque.

Les articles de ce numéro donnent à voir quelques scènes et pratiques sociales à travers lesquelles se construisent et se déploient ce que l'on peut appeler des "paysages mémoriels » (memoryscape) dans le sens donné à ce terme par Zsuzsa Gille, pour qui il désigne moins des sites géographiques qui comportent des monuments ou des objets mémoriaux qu'un « panorama de pratiques mémorielles qui se croisent et interagissent à différentes échelles ${ }^{34} »$.

\section{Objets mémoriels et scènes performatives de mémoire dans les sociétés contemporaines des Balkans}

À partir d'une recherche sur les communautés religieuses grecques du Mont Athos, Isabelle Dépret met en avant un des lieux majeurs dans lesquels la globalisation des sociétés balkaniques s'accompagne de discours sur la mémoire : la scène patrimoniale. I. Dépret montre que la labellisation du Mont Athos par l'UNESCO en 1988 et sa reconnaissance par l'État grec comme un "lieu de mémoire national » entraînent des tensions dans la gestion de ce patrimoine entre l'État et les communautés religieuses athonites. Ces dernières s'opposent à une tendance à la sécularisation du patrimoine et du lieu, et à ce que des professionnels du patrimoine deviennent des gestionnaires et décideurs de la qualité patrimoniale et mémorielle du Mont Athos. Cette situation de tension est une occasion pour la communauté religieuse de formuler des discours sur la tradition et sur la mémoire. Ces discours prônent, d'une part, la tradition millénaire d'autogestion de ce lieu et, d'autre part, la figure des moines comme des «monuments vivants d'un paysage physique et moral, d'un passé immuable qu'ils contribueraient activement à sauvegarder ».

Alors que certains discours sur la tradition et sur la mémoire athonite semblent, à première vue, partagés par les différents moines des vingt monastères du Mont Athos, l'auteure montre que ces discours sont mobilisés pour justifier les attitudes finalement différentes, voire opposées, adoptées par les monastères par rapport à la question du tourisme et du monde numérique dans ce lieu sacré et, plus généralement, par rapport à la globalisation.

L'apport de cet article dans l'économie générale du numéro repose avant tout sur la mise en avant des échelles variées (locales, régionales, nationales, internationales) de constitution et de déploiement des métamémoires dans les sociétés balkaniques. En plus des « arènes mémorielles » locales et nationales, I. Dépret montre que les discours 
sur la tradition et la mémoire du Mont Athos s'élaborent aussi par des négociations lors des commémorations en Macédoine ou par la présence massive de la Russie au Mont Athos, ce qui met en lumière un travail sur le passé articulant le religieux, le politique et la géopolitique régionale.

Gilles de Rapper explore une autre scène publique majeure où se construit le discours mémoriel aujourd'hui, à savoir la mémoire du communisme et en particulier la production photographique de cette période. À partir d'une étude ethnologique menée en Albanie sur la production récente des albums de photographie de cette époque, l'auteur analyse les conditions et les enjeux de cette production photographique durant le régime communiste et aujourd'hui.

L'auteur montre que la photographie est une scène performative pour la mémoire à plusieurs titres. Tout d'abord, la photographie n'est pas simplement le reflet d'une réalité sociale ayant une valeur de documentation de la vie sociale de l'époque communiste. Durant ce régime, elle a surtout été un moyen pour faire advenir une réalité, voulue et imaginée par le pouvoir, la photographie participant activement, à côté d'autres moyens visuels, à la fabrication de la "nouvelle société ». L'auteur parle en ce sens des "cadres photographiques de la mémoire ", allusion aux " cadres sociaux de la mémoire » de Halbwachs, pour montrer que la photographie avait cette fonction importante de «délimiter ce dont il fallait se souvenir et comment il fallait se souvenir ». C'est aussi le cas de nos jours de ces albums photographiques puisque la «mise en scène et en circulation de ces photographies dans le contexte d'aujourd'hui constitue un acte mémoriel et une performance qui disent autre chose que des faits historiques ".

Alors que les photographes-interlocuteurs du chercheur sont préoccupés à montrer le caractère de vérité du matériel visuel, en omettant leur participation active à la définition d'une réalité et d'une "mémoire du communisme", on peut se demander dans quelle mesure l'enquête scientifique joue un rôle dans la production de cette mémoire. Dans ce sens, l'objectif de l'enquête ne serait pas seulement de montrer des mécanismes de production de cette «réalité» (par la photographie), mais de comprendre et de mettre en lumière comment le dispositif scientifique participe lui aussi à cette entreprise, à côté de la photographie.

La question du dispositif d'enquête se pose également pour l'article d'Adriana Cupcea, même si elle n'y apparaît pas de façon explicite. Dans son étude sur la communauté rom musulmane turcophone de Medgidia (Roumanie), l'auteure aborde la mémoire par sa réactualisation sous la forme des récits de vie suscités par l'histoire orale et par l'enquête de terrain. Ces discours sur le passé, considérés par A. Cupcea comme des récits mémoriels, constituent des actes de production d'une identité et d'une mémoire de groupe dans des conditions d'absence d'archives et de sources écrites sur la constitution de cette communauté. La mémoire, sous la forme des souvenirs évoqués de la période du communisme, surgit dans ce passage douloureux de l'après 1989 , un temps de détresse synonyme pour les membres de cette communauté de la fin d'un âge d'or; il renvoie à un déclassement social et à une précarité économique à travers la perte d'une identité ouvrière qui donnait à ces personnes une reconnaissance au sein de la société et une place à l'intérieur d'une communauté locale. L'après 1989 renvoie en outre à la perte d'une unité familiale, par les migrations à l'étranger ou régionales que cette période a engendrées, et à la disparition des solidarités sociales plus généralement. L'auteure montre que la perte d'une identité sociale, ouvrière et locale 
entraîne pour ces populations un retour à des catégorisations ethniques plutôt stigmatisantes (Roms) et un renforcement de l'identité religieuse, d'une part; et que, d'autre part, cette situation engendre la production par les membres de la communauté d'un discours nostalgique. Cette orientation nostalgique va dans le sens d'une quête de valorisation par une (sur)identification à la communauté turque et par un discours sur les racines, lesquels performent une mémoire historique autour de l'héritage ottoman.

Ces récits de type mémoriel sur la période communiste suscités, entre autres, par le cadre de l'enquête de terrain, posent donc à nouveau la question de la place du dispositif de recherche dans la production d'une mémoire de groupe. Cet enjeu est important dans les conditions de l'absence de documents d'archive, le dispositif scientifique pouvant fonctionner comme une scène performative de la mémoire historique de la communauté et donc comme participant à la fabrication de la communauté.

41 Vintilă Mihăilescu met en lumière d'autres dispositifs et pratiques où des mémoires sont mobilisées, cette fois-ci autour des produits gastronomiques « traditionnels » et de la recherche du " goût du 'vrai' ». La première partie de l'article aborde les différents facteurs et les étapes intervenus dans la fabrication des produits "traditionnels » (le fondement domestique de cette cuisine, sa nationalisation, la globalisation de l'alimentation, les marchés alternatifs des élites urbaines). La question de la mémoire intervient dans la dernière partie de l'article, à partir de l'interrogation suivante : alors que les produits « traditionnels » reposent sur un processus classique d'invention et de réinvention des traditions, sur quoi repose cette croyance partagée qui fait que les individus semblent être plutôt d'accord sur le fait qu'un produit est (ou n'est pas) «traditionnel »? La réponse suggérée par l'auteur fait référence à un phénomène de type mémoriel. L'auteur fait l'hypothèse que la possibilité même de revendiquer une authenticité des produits et un «retour au vrai goût» implique qu'il existe des souvenirs réels de cette cuisine "de grand-mère ", «d'antan » ou " authentique » et qu'il y ait un consensus autour de ces souvenirs. Cependant, les souvenirs relatifs à cette cuisine intime ne sont jamais réellement partagés; ils sont, dans ce sens, plus individuels que collectifs. En revanche, ce qui est partagé, c'est plutôt une métamémoire du "traditionnel ", c'est-à-dire un discours sur cette mémoire. Il existerait ainsi une sorte de "pacte mémoriel» conclu entre producteurs et consommateurs, pour le coup très performatif, qui ferait que tout le monde s'accorde sur l'existence d'une cuisine "traditionnelle», une valeur en soi qui devrait être de retour.

V. Mihăilescu met en avant les différentes scènes qui permettent la construction de cette métamémoire du "goût du 'vrai' " (foires et festivals, médias et scènes publicitaires) et les spectacles «collectifs, répétitifs et corporels » qui la performent. L'auteur affirme que ces mises en spectacle des produits ne sont pas une mise en lumière d'une mémoire déjà là, mais des ritualités qui deviennent "des bonnes occasions de mémorisation et d'apprentissage de la mémoire ».

43 Enfin, l'article de Biborka Ádám aborde les commémorations liées à deux fêtes nationales en Roumanie, fêtes qui ont cristallisé à travers le temps les relations conflictuelles entre les communautés roumaine et hongroise dans le Pays Sicule, en Transylvanie. Le texte suggère que ces fêtes sont des cérémonies au cours desquelles une mémoire est réactualisée et performée dans le présent. La mémoire du groupe (mémoire culturelle au sens de Jan Assmann) participe à la construction d'une identité 
nationale en contexte minoritaire et reconduit la production des frontières ethniques de type Nous/Eux. C'est également une mémoire historique de la cohabitation roumano-hongroise qui est réactualisée et performée à travers ces cérémonies. Les notions de "mémoire historique " et de "postmémoire " peuvent être convoquées dans cet article car elles permettent de comprendre la ferveur transgénérationnelle liée à de tels événements et certaines tensions en Transylvanie ${ }^{35}$, alors même que les derniers témoins directs des événements fondateurs de cette mémoire commencent à disparaître.

\section{NOTES}

1. OLICK Jeffrey, RoBBINS Joyce, « Social Memory Studies: from 'Collective Memory' to the Historical Sociology of Mnemonic Practices", Annual Review of Sociology, vol. 24, 1998, p. 105-140 ; OLICK Jeffrey, « Between Chaos and Diversity: Is Social Memory Studies a Field? », International Journal of Politics, Culture and Society, vol. 22, n 2, 2009, p. 249-252.

2. Mentionnons quelques moments marquants dans ce sens : l'entrée « Mémoire collective » dans LE GOFF Jacques, CHARTIER Roger, REVEL Jacques (dir.), La Nouvelle histoire, Paris, Retz, 1978 ; NORA Pierre, Les lieux de mémoire, vol. Is La République, Paris, Gallimard, 1984 et en particulier le texte d'ouverture intitulé « Entre mémoire et histoire».

3. Voir par exemple MIHAILESCU Vintilă, «Introducere » [Introduction], dans Vintilă Mihăilescu (dir.), Etnografii urbane. Cotidianul văzut de aproape [Ethnographies urbaines. Le quotidien vu de près], Iaşi, Polirom, 2009, p. 8-12.

4. VULTUR Smaranda, Negotiating the Past or on the Way in which Trauma Is Approached in the Discourse of the Former Deportees to the Field of Bărăgan (1951-1956), 2011, en ligne: http:// anciensiteusagespublicsdupasse.ehess.fr/fileadmin/PDFarticles/

Vultur._Negotiating_the_Past.pdf (consulté en mars 2020)

5. Rousso Henri, "Vers une mondialisation de la mémoire ", Vingtième siècle. Revue d'histoire, vol. 2, no 94, 2007, p. 3-10.

6. Ibid. p. 5 .

7. Rousso, « Vers une mondialisation de la mémoire », op. cit., p. 6.

8. Pour noter seulement quelques études menées explicitement sur les questions mémorielles dans ce paysage thématique vaste, voir par exemple: BROWN Keith (dir.), Homelands in Question: Paradoxes of Memory and Exil in South-Eastern Europe, Balkanologie, vol. V, n ${ }^{\circ} 1-2,2001$; MAYER Françoise (dir.), Mémoires à l'Est, Revue d'études comparatives Est-Ouest, vol. 37, nº 3, 2006 ; BUCUR Maria, Heroes and Victims: Remembering War in Twentieth-Century Romania, Bloomington (IN), Indiana University Press, 2009 ; ToDorova Maria, GILlE Zsuzsa (eds), Post-Communist Nostalgia, New York, Oxford, Berghahn Books, 2010 ; valtchinova Galia (dir.), Paysages religieux et mémoriels: visions, pratiques et politiques, Études balkaniques, ${ }^{\circ} 18,2011$; ВОТЕA Bianca, Territoires en partage. Politiques du passé et expériences de cohabitation en Transylvanie, Paris, Petra, 2013 ; BECHTEL Delphine, JURGENSON Luba, (dir.), Le Tourisme mémoriel en Europe centrale et orientale, Paris, Petra, 2013 ; LosonCZY Anne-Marie, « 'Arpenter la patrie retrouvée'. Tourisme mémoriel et tourisme identitaire post-communistes en Transcarpathie", Civilisations. Revue internationale d'anthropologie et de sciences humaines, $\mathrm{n}^{\circ} 63,2014$, p. 223-236 ; ANTON Lorena, « ‘On n'en parlera jamais...'. Interroger la 
mémoire de l'avortement en Roumanie du communisme ", Ethnologie française, vol. 44, $\mathrm{n}^{\circ} 3,2014$, p. 421-428 ; PALMBERGER Monika, How Generations Remember Conflicting Histories, Londres, Palgrave, 2017 ; DE RAPPER Gilles, « Photography and Remembrance », dans Jonila Godole, Idrit Idrizi (dir.), Between Apathy and Nostalgia. Private and Public Recollections of Communism in Contemporary Albania, Tirana, IDMC, 2019, p. 103-119.

9. PERONI Michel, BELKIS Dominique, «Pragmatique de la mémoire et enquête sur les régimes de mémorialité », EspacesTemps.net, Traverses, 2015, en ligne: https://www.espacestemps.net/ articles/pragmatique-de-la-memoire-et-enquete-sur-les-regimes-de-memorialite/ (consulté en septembre 2020).

10. HALBWACHS Maurice, Les cadres sociaux de la mémoire, Paris, Albin Michel, 1997 [1925] et La mémoire collective, Paris, Albin Michel, 1994 [1950].

11. POUILLON Jean, «La tradition : transmission ou reconstruction?", dans Jean Pouillon, Fétiches sans fétichisme, Paris, Maspero, 1975, p. 155-173.

12. AUSTIN John, How to Do Things With Words, conférences données en 1955 réunies dans une publication posthume en 1962, avec la traduction en français : Quand dire c'est faire, Paris, Éditions du Seuil, 1970.

13. Jonathan Culler commente le sens de "performatif» donné par Austin et l'apport de cette perspective: CULLER Jonathan, "Philosophie et littérature. Les fortunes du performatif», Littérature, vol. 4, $\mathrm{n}^{\circ}$ 144, 2006, p. 81-100.

14. wULF Christoph, Anthropology: A Continental Perspective, Chicago, The University of Chicago Press, 2013, p. 206 (voir notamment le chapitre « Theories and Practices of Performative »).

15. Pour une analyse de l'approche performative du genre de Judith Butler, voir CULLER Jonathan, op. cit., BUTLER Judith, Troubles dans le genre, Paris, La Découverte, 2005 et Bodies that Matter, Londres, New York, Routledge, 1993.

16. DE CERTEAU Michel, L'invention du quotidien, I. Arts de faire, Paris, Seuil, 1980.

17. ROACH Joseph, "Culture and Performance in the Circum-Atlantic Worlds ", dans Erin Striff (dir.), Performance Studies, New York, Palgrave, 2003, p. 124-137, ici p. 125 (notre traduction).

18. WULF, Anthropology, op. cit., p. 205.

19. RICceUR Paul, La Mémoire, l'histoire, l'oubli, Paris, Seuil, 2000, p. 67.

20. CANDAU Joël, Anthropologie de la mémoire, Paris, Armand Colin, 2005, p. 78-82.

21. PERONI, BELKIS, «Pragmatique de la mémoire", op. cit. Les différents articles de ce numéro mettent en avant, de manière explicite ou implicite, la dimension performative des mémoires.

22. CONNERTON Paul, How Societies Remember, Cambridge, Cambridge University Press, 1989, p. 5 (notre traduction).

23. Ibid. (notre traduction).

24. PlATE Liedeke, SMELIK Anneke, "Performing Memory in Art and Popular Culture. An Introduction ", dans Liedeke Plate, Anneke Smelik (dir.), Performing Memory in Art and Popular Culture, New York, Oxfordshire, Routledge, 2013, p. 1-22.

25. Ibid., p. 3-4.

26. Ibid., p. 6

27. C'est le propos d'un éditorialiste américain repris par Joseph Roach (op. cit.).

28. Comme nous l'avons mentionné auparavant en citant Halbwachs, la notion de mémoire individuelle est à prendre avec précaution, puisque toute mémoire individuelle est une mémoire sociale.

29. Nous pouvons faire la distinction entre mémoire sociale et mémoire collective : si la mémoire a un caractère social puisque, comme le disait Halbwachs, pour se rappeler nous avons besoin d'autres individus, cela n'induit pas obligatoirement l'existence des mémoires collectives, autrement dit des contenus mémoriels partagés par tous les individus. Marie-Claire Lavabre distingue deux approches différentes de la notion de "mémoire collective » dans le travail de 
Halbwachs (bien que l'auteur ne pose pas cette distinction de manière explicite). Dans une certaine mesure, ces deux approches correspondent à notre distinction entre la mémoire collective et la mémoire sociale. Lavabre note que, dans une première conception holiste de la "mémoire collective ", le groupe apparaît comme déjà constitué, comme une entité en soi dont les membres possèdent une mémoire en commun. Selon une deuxième approche, « la mémoire serait collective non pas parce qu'elle est la mémoire du groupe en tant que groupe, mais parce que le collectif, le social, est l'état dans lequel existent les individus» (LAVABRE Marie-Claire, "Usages et mésusages de la notion de mémoire ", Critique internationale, $\mathrm{n}^{\circ} 7,2000$, p. 55).

30. WULF, Anthropology, op. cit., p. 206.

31. HALBWACHS, Les cadres sociaux de la mémoire et La mémoire collective, op. cit.; BOYM Svetlana, The Future of Nostalgia, New York, Basic Books, 2001 ; TODOROVA, GILlE, Post-Communist Nostalgia, op. cit.

32. Dans une recherche longitudinale, Michèle Leclerc-Olive montre que ses interlocuteurs, interrogés de manière répétée à distance de plusieurs années sur une même expérience de leur histoire, attribuent une valeur différente à celle-ci selon les fluctuations intervenues dans leur vie, comme par exemple leur carrière sociale ou d'autres transformations de leur situation de vie (LECLERC-OLIVE Michèle, "Au-delà des épistémologies sédentaires. Les changements urbains au miroir de l'exil», Parcours anthropologiques, $\mathrm{n}^{\circ} 10,2015$, en ligne: https:// journals.openedition.org/pa/443 (consulté en mars 2020).

33. RIC@EUR Paul, « Entre mémoire et histoire », Projet. Mémoires des peuples, $\mathrm{n}^{\circ} 248,1996$.

34. TODOROVA, GILlE, Post-Communist Nostalgia, op. cit., p. 288 (notre traduction).

35. BOTEA, Territoires en partage, op. cit.

INDEX

Index géographique : Balkans

\section{AUTEURS}

BIANCA BOTEA

Université Lumière Lyon 2, Laboratoire d'anthropologie des enjeux contemporains bianca.botea[at]univ-lyon2.fr

\section{VINTILĂ MIHĂILESCU}

École nationale d'études administratives et politiques, Bucarest 